\title{
Range of forces on host-metal atoms around interstitial hydrogen in Pd and $\mathrm{Nb}$
}

\author{
C. Elsässer, M. Fähnle, and L. Schimmele \\ Max-Planck-Institut für Metallforschung, Institut für Physik, Heisenbergstrasse 1, D-70569 Stuttgart, Germany \\ C. T. Chan and K. M. Ho \\ Ames Laboratory and Department of Physics, Iowa State University, Ames, Iowa 50011
}

(Received 7 March 1994; revised manuscript received 9 May 1994)

\begin{abstract}
Forces on the host-metal atoms surrounding a hydrogen atom at different interstitial positions in palladium and niobium are calculated from first principles in the local-density approximation with a mixed-basis pseudopotential supercell approach. For supercells $\operatorname{Pd}_{n} \mathrm{H}(n=1,4,8,16,32)$ previously reported results are reanalyzed and corrected based on a recently noticed simplification in the computational method. Results are presented for supercells $\mathrm{Nb}_{n} \mathrm{H}(n=1,2,4,8,16)$. In both elements the displacement forces decay dominantly within the range of one lattice constant. The elastic force-dipole tensors are determined from the first-principles forces. The results for octahedral occupation of $\mathrm{H}$ in $\mathrm{Pd}$ and tetrahedral occupation of $\mathrm{H}$ in $\mathrm{Nb}$ support the picture of static occupation of distinct interstitial sites.
\end{abstract}

For the understanding of many properties of metalhydrogen systems information about the microscopic interaction between the interstitial hydrogen atom and the surrounding host-metal atoms is essential. A wide range of materials has been investigated, under the viewpoints of applied technologies such as hydrogen-energy storage as well as of basic research, with the aim of understanding microscopic properties such as interstitial site locations, vibrations, and diffusion of light particles in a transitionmetal host. ${ }^{1,2}$ Two of the best known materials are the $\mathrm{Pd}-\mathrm{H}$ and $\mathrm{Nb}-\mathrm{H}$ systems. However, in spite of much information accumulated in the literature, there are still open questions worthy of further study.

One of these questions is concerned with the range of forces on or displacements of the host-metal atoms surrounding the interstitial hydrogen atom. ${ }^{3,4}$ For simplicity it is most favorable to imagine that the forces have a short range from the $\mathrm{H}$ atom to only the nearest- or nextnearest-neighbor metal atoms and that the action of the impurity is well screened from further metal atoms by the conduction electrons. However, this is an assumption which requires microscopic support.

Experimentally, the direct measurement of atomic forces or displacements is difficult. The first ingredient to get the forces is the knowledge of the preferred location of light particles in the metal lattice. This can be rather well determined, for instance, with the ion-channeling method: ${ }^{5}$ In the face-centered-cubic (fcc) $\mathrm{Pd}$ and the body-centered-cubic (bcc) $\mathrm{Nb}$, the $\mathrm{H}$ atoms occupy octahedral and tetrahedral sites, respectively.

Next the directions of forces and relaxation patterns of the metal atoms are given by the local symmetry of the interstitial sites. The field of the forces can be expanded in multipoles, ${ }^{3,6,7}$ starting with the second-rank forcedipole tensor $\mathcal{P}$ with the components

$$
P_{i j}=\sum_{m} f_{j}^{m} x_{i}^{m}
$$

where the $x_{i}^{m}$ and $f_{j}^{m}(i, j=1,2,3)$ are the $i$ th Cartesian coordinate and the $j$ th Cartesian Kanzaki-force component, respectively, on the $m$ th metal atom. The $\mathrm{H}$ atom is located at the origin of the coordinate system. The force-dipole tensor is the connecting quantity between the atomistic and the elastic-continuum description of lattice distortions due to point defects in crystals. ${ }^{7}$ The number of independent tensor components is limited by the local interstitial symmetry. For the octahedral and tetrahedral sites $O$ and $T$, with cubic symmetry in $\mathrm{Pd}$ and for the triangular saddle point $S$ between them, the tensor $\mathcal{P}$ is given by

$$
\mathcal{P}=\left(\begin{array}{lll}
A & C & C \\
C & A & C \\
C & C & A
\end{array}\right)
$$

with $C=0$ for $O$ and $T$. In $\mathrm{Nb} \mathcal{P}$ is given by

TABLE I. Corrected absolute values of forces on Pd atoms at different neighbor shells around an octahedral $\mathrm{H}$ atom in $\mathrm{Pd}_{n} \mathrm{H}$ (in Ry/a.u.): $d / a$ is the distance between $\mathrm{Pd}$ and $\mathrm{H}$ atoms in units of the calculated equilibrium lattice constant of pure $\mathrm{Pd}, a=a_{0}(\mathrm{Pd})=7.33$ a.u. $=3.88 \AA$. Values marked by an asterisk indicate that these metal atoms are located on the Wigner-Seitz cell boundary of the hydrogen sublattice.

\begin{tabular}{ccccc}
\hline \hline & \multicolumn{4}{c}{$\mathrm{H}$ at $O$ in Pd } \\
Shell & $(1)$ & $(2)$ & $(3)$ & $(4)$ \\
\hline$\frac{d}{a}$ & $\frac{1}{2}$ & $\frac{\sqrt{3}}{2}$ & $\frac{\sqrt{5}}{2}$ & $\frac{3}{2}$ \\
$\mathrm{Pd}$ & $0.0^{*}$ & & & \\
$\mathrm{Pd}_{4} \mathrm{H}$ & $0.0^{*}$ & $0.0^{*}$ & & \\
$\mathrm{Pd}_{8} \mathrm{H}$ & 0.013 & $0.0^{*}$ & & \\
$\mathrm{Pd}_{16} \mathrm{H}$ & 0.010 & $0.0^{*}$ & $0.0^{*}$ & \\
$\mathrm{Pd}_{32} \mathrm{H}$ & 0.009 & 0.006 & $0.001^{*}$ & $0.000^{*}$ \\
\hline \hline
\end{tabular}




$$
\mathcal{P}=\left(\begin{array}{ccc}
A & C & 0 \\
C & A & 0 \\
0 & 0 & B
\end{array}\right)
$$

for the tetrahedral and octahedral sites $T$ and $O$, with tetragonal symmetry, and for the saddle point $S$ on the straight line between two neighboring tetrahedral sites. $C=0$ for $T$ and $O$ again. One characteristic quantity of the force-dipole tensor is its trace $\operatorname{Tr} \mathcal{P}=3 A$ for $\mathrm{H}$ in $\operatorname{Pd}$ and $\operatorname{Tr} \mathcal{P}=2 A+B$ for $\mathrm{H}$ in $\mathrm{Nb}$, which is proportional to the $H$-induced isotropic volume expansion of the metal. Another quantity is the measure for tetragonal anisotropy, conventionally given by the dimensionless expression $\frac{3|A-B|}{2 A+B}$. The nonzero off-diagonal components $C$ are due to the trigonal and orthorhombic local symmetry at the saddle point in $\mathrm{PdH}$ and $\mathrm{NbH}$, respectively.

The components of the force-dipole tensor can be measured via diffraction and scattering of $\mathrm{x}$ rays. ${ }^{1}$ To get an estimate for the forces $f_{i}^{m}$ from the measured tensor components $A$ and $B$ an assumption is necessary for the range of nonvanishing forces in the summation $m$ over host-metal atoms. It is very common in bcc metals to sum over the first two neighbor shells. ${ }^{4}$ The inclusion of at least the second shell is essential for $\mathrm{H}$ in $\mathrm{Nb}$ in the static picture to achieve agreement with the experimental finding of an almost isotropic (cubic) force-dipole tensor $\left[\frac{3|A-B|}{2 A+B} \leq 0.06\right.$ (Ref. 4)].

Recently Dosch et al. ${ }^{8}$ have questioned the simple picture of $\mathrm{H}$ atoms statically occupying individual interstitial sites in bcc metals. They propose a dynamical model based on the assumption that lattice distorsions cannot immediately follow $\mathrm{H}$ atoms rapidly jumping between neighboring tetrahedral sites in $\mathrm{Nb}$. The range of static forces is assumed to be limited to the nearestneighbor $\mathrm{Nb}$ atoms, but the dynamics of the $\mathrm{H}$ atom leaves sufficient flexibility of the model to obtain a better quantitative agreement with their diffuse-scattering spectra than the static model, including the vanishing anisotropy of the force-dipole tensor.

The dynamical picture was supported theoretically by Wahnström and $\mathrm{Li}^{9}$ In their molecular-dynamics simulations they found very rapid local jumps of the $\mathrm{H}$ atoms between neighboring tetrahedral sites. These motions are confined mainly to the so-called $4 T$ configurations, whose local symmetry is equal to the one of octahedral sites, and less to the $3 T$ configurations with a lower symmetry suggested by Dosch et al. ${ }^{8}$
TABLE II. Corrected absolute values of forces on Pd atoms around a tetrahedral $\mathrm{H}$ atom in $\mathrm{Pd}_{n} \mathrm{H}$ (in $\mathrm{Ry} / \mathrm{a}$.u.). See the caption of Table I.

\begin{tabular}{ccccc}
\hline \hline & \multicolumn{4}{c}{$\mathrm{H}$ at $T$ in $\mathrm{Pd}$} \\
Shell & $(1)$ & $(2)$ & $(3)$ & $(4)$ \\
\hline$\frac{d}{a}$ & $\frac{\sqrt{3}}{4}$ & $\frac{\sqrt{11}}{4}$ & $\frac{\sqrt{19}}{4}$ & $\frac{3 \sqrt{3}}{4}$ \\
$\mathrm{PdH}$ & $0.0^{*}$ & & & \\
$\mathrm{Pd}_{4} \mathrm{H}$ & 0.065 & & & \\
$\mathrm{Pd}_{8} \mathrm{H}$ & 0.066 & $0.000^{*}$ & & \\
$\mathrm{Pd}_{16} \mathrm{H}$ & 0.066 & 0.006 & & \\
$\mathrm{Pd}_{32} \mathrm{H}$ & 0.069 & 0.005 & 0.000 & 0.001 \\
\hline \hline
\end{tabular}

In the present work we report first-principles supercell calculations in the local-density approximation ${ }^{10}$ for the forces on the metal atoms on ideal lattice sites surrounding one interstitial $\mathrm{H}$ atom per supercell in $\mathrm{Pd}$ and $\mathrm{Nb}$. Forces in cubic $\mathrm{Pd}_{n} \mathrm{H}$ supercells $(n=1,4,8,16,32)$ have been reported in our earlier work. ${ }^{11}$ However, based on a recently noticed important simplification ${ }^{12,13}$ in our computational method for the forces, the values of these data were found to be in part inadequate. In the following the reanalyzed forces are reported and the components of the resulting force-dipole tensors for three different interstitial sites are compared with experimental results. Results for forces in cubic $\mathrm{Nb}_{n} \mathrm{H}$ supercells $(n=1,2,4,8,16)$ and the related force-dipole tensors are given subsequently.

A microscopic calculation of the force-dipole tensor for $\mathrm{H}$ in the fcc metals $\mathrm{Pd}$ and $\mathrm{Pt}$ in the framework of the effective-medium theory has been reported recently. ${ }^{14}$ Our first-principles results confirm those result for $\mathrm{Pd}$ and support the appropriateness of the effective-medium theory. For $\mathrm{H}$ in the bcc metal $\mathrm{Nb}$ we are not aware of any earlier microscopic theoretical determination of the force-dipole tensor.

It is important to be aware that the forces calculated from first principles in the supercells containing one $\mathrm{H}$ atom strictly are not the Kanzaki forces $f_{j}^{m}$. These are defined as those forces which have to be applied to the atoms in the pure metal without $\mathrm{H}$ in order to cause the same atomic displacements as if the disturbing $\mathrm{H}$ atom were present. In our calculations the forces of the $\mathrm{H}$ atoms on the metal atoms at the ideal lattice positions are evaluated. Hence small contributions to the Kanzaki forces resulting from changes in the force-constant matrix of the metal are neglected [cf. Eq. (2.8) in Ref. 15].

TABLE III. Corrected absolute values of forces on a $\mathrm{H}$ atom located at a triangular saddle point in $\mathrm{Pd}_{n} \mathrm{H}$ and on the $\mathrm{Pd}$ atoms around it (in Ry/a.u.). See the caption of Table I.

\begin{tabular}{|c|c|c|c|c|c|c|c|c|c|c|c|}
\hline & & & & & $\mathrm{H}$ at & $\mathrm{Pd}$ & & & & & \\
\hline Shell & $H$ & (1) & (2) & (3) & $(4)$ & $(5)$ & $(6)$ & $(7)$ & $(8)$ & (9) & (10) \\
\hline$\frac{d}{a}$ & 0 & $\frac{\sqrt{6}}{6}$ & $\frac{\sqrt{3}}{3}$ & $\frac{\sqrt{2}}{2}$ & $\frac{\sqrt{6}}{3}$ & $\frac{\sqrt{30}}{6}$ & 1 & $\frac{\sqrt{42}}{6}$ & $\frac{2 \sqrt{3}}{3}$ & $\frac{\sqrt{6}}{2}$ & $\frac{\sqrt{66}}{6}$ \\
\hline $\mathrm{PdH}$ & 0.0 & $0.0^{*}$ & & & & & & & & & \\
\hline $\mathrm{Pd}_{4} \mathrm{H}$ & 0.002 & 0.098 & 0.014 & & & & & & & & \\
\hline $\mathrm{Pd}_{8} \mathrm{H}$ & 0.001 & 0.100 & 0.015 & 0.005 & $0.000^{*}$ & & & & & & \\
\hline $\mathrm{Pd}_{16} \mathrm{H}$ & 0.000 & 0.100 & 0.020 & 0.009 & 0.005 & 0.003 & & & & & \\
\hline $\mathrm{Pd}_{32} \mathrm{H}$ & 0.000 & 0.105 & 0.020 & 0.009 & 0.006 & 0.003 & 0.002 & 0.001 & 0.001 & 0.001 & 0.001 \\
\hline
\end{tabular}


TABLE IV. The force-dipole tensor component $A$ and its contributions from the different neighbor shells of $\mathrm{Pd}$ atoms around an octahedral $\mathrm{H}$ atom in $\mathrm{Pd}_{n} \mathrm{H}$ (in $\mathrm{eV}$ ).

\begin{tabular}{lccccc}
\hline \hline & \multicolumn{7}{c}{$\mathrm{H}$ at $O$ in $\mathrm{Pd}$} \\
Shell & $(1)$ & $(2)$ & $(3)$ & $(4)$ & $A$ \\
\hline $\mathrm{PdH}$ & 0.0 & & & & 0.0 \\
$\mathrm{Pd}_{4} \mathrm{H}$ & 0.0 & 0.0 & & & 0.0 \\
$\mathrm{Pd}_{8} \mathrm{H}$ & 1.30 & 0.0 & & & 1.30 \\
$\mathrm{Pd}_{16} \mathrm{H}$ & 0.98 & 0.0 & 0.0 & & 0.98 \\
$\mathrm{Pd}_{32} \mathrm{H}$ & 0.94 & 1.38 & -0.40 & -0.13 & 1.79 \\
\hline \hline
\end{tabular}

In supercell calculations of absolute values of the force acting on the $m$ th metal atom

$$
\left|\mathbf{f}^{m}\right|=\sqrt{\sum_{j=1}^{3}\left(f_{j}^{m}\right)^{2}}
$$

and components $P_{i j}$ of the force-dipole tensor from the Cartesian components $x_{i}^{m}$ and $f_{j}^{m}$ one has to check carefully whether the $m$ th metal atom is located on a boundary wall of the hydrogen-sublattice Wigner-Seitz cell. In this case the force components perpendicular to the wall vanish identically by symmetry. In comparison with forces expected around an isolated $\mathrm{H}$ atom the components parallel to the wall may be reduced as well by superposition. Additionally the value of such a force component is to be divided by the number of Wigner-Seitz cells sharing the atom on a common wall. This ensures the correct symmetry weight of the force in the summation over all equivalent metal atoms of a neighbor shell for the calculation of the force-dipole tensor. In Tables IIII and VII-IX such forces are indicated by an asterisk. For the forces on the atoms located within the cell the effects of superposition can only be reduced by increasing the supercell size.

For the calculation of the forces on the metal atoms in $\mathrm{Pd}_{n} \mathrm{H}$ supercells $(n=1,4,8,16,32)$ from first principles a mixed-basis pseudopotential method ${ }^{16-18}$ has been used. The formalism and the computational details are given in Refs. 11 and 19. Recently ${ }^{12,13}$ it was found that one contribution to the forces, originating from changes in the fractional occupations of the one-electron Bloch states, exactly cancels out because of the variational property of the particular energy functional used in these calculations. This contribution [cf. the last sum in Eq. (17) of

TABLE V. The force-dipole tensor component $A$ and its contributions from the different neighbor shells of $\mathrm{Pd}$ atoms around an tetrahedral $\mathrm{H}$ atom in $\mathrm{Pd}_{n} \mathrm{H}$ (in $\mathrm{eV}$ ).

\begin{tabular}{lccccc}
\hline \hline & \multicolumn{7}{c}{$\mathrm{H}$ at $T$ in $\mathrm{Pd}$} \\
Shell & $(1)$ & $(2)$ & $(3)$ & $(4)$ & $A$ \\
\hline $\mathrm{PdH}$ & 0.0 & & & & 0.0 \\
$\mathrm{Pd}_{4} \mathrm{H}$ & 3.75 & & & & 3.75 \\
$\mathrm{Pd}_{8} \mathrm{H}$ & 3.77 & 0.02 & & & 3.79 \\
$\mathrm{Pd}_{16} \mathrm{H}$ & 3.82 & 1.81 & & & 5.63 \\
$\mathrm{Pd}_{32} \mathrm{H}$ & 3.98 & 1.80 & -0.11 & -0.17 & 5.50 \\
\hline \hline
\end{tabular}

TABLE VI. Traces $\operatorname{Tr} \mathcal{P}=3 A$ and nonzero off-diagonal components $C$ (in $\mathrm{eV}$ ) of the force-dipole tensors for $\mathrm{H}$ at the octahedral and tetrahedral sites and the saddle point in Pd. Values in parentheses contain force contributions from shells with $d / a>1$.

\begin{tabular}{lcccc}
\hline & $\mathrm{H}$ at $O$ & $\mathrm{H}$ at $T$ & \multicolumn{2}{c}{$\mathrm{H}$ at $S$} \\
& $\operatorname{Tr} \mathcal{P}$ & $\operatorname{Tr} \mathcal{P}$ & $\operatorname{Tr} \mathcal{P}$ & $C$ \\
\hline $\mathrm{Pd}_{8} \mathrm{H}$ & 3.9 & 11.4 & 14.0 & -1.6 \\
$\operatorname{Pd}_{16} \mathrm{H}$ & 2.9 & 16.9 & 18.3 & -1.3 \\
$\mathrm{Pd}_{32} \mathrm{H}$ & $7.0(5.4)$ & $17.3(16.5)$ & $19.4(18.4)$ & $-1.2(-1.4)$ \\
\hline \hline
\end{tabular}

Ref. 19] was taken into account approximately in Ref. 11 in the erroneous expectancy to improve the accuracy of the forces. It turned out to change the values of the forces by less than $10^{-2} \mathrm{Ry} / \mathrm{a} . \mathrm{u}$. $(1 \mathrm{Ry}=13.6 \mathrm{eV}, 1$ a.u. $=0.529 \AA$ ), which is negligible for many cases, but now we noticed that it became significant in the case of rather small forces in the $\mathrm{Pd}_{n} \mathrm{H}$ supercells. In particular, it did not decrease with increasing distance from the $\mathrm{H}$ atom, suggesting a long range of the distortion field. The result of Refs. 12 and 13, that these force contributions vanish exactly, caused us to reanalyze our force results in $\mathrm{Pd}_{n} \mathrm{H}$ by omitting the corresponding term.

The corrected data are given in Tables I-III for $\mathrm{H}$ at the three sites $O, T$, and $S$ (they replace the less accurate data given in the Tables 3-5 of Ref. 11). The forces decay within the range of the $\mathrm{Pd}_{32} \mathrm{H}$ supercells below $10^{-3}$ Ry/a.u., which is less than our estimated limit of accuracy of about $5 \times 10^{-3} \mathrm{Ry} / \mathrm{a} . \mathrm{u}$. Furthermore, the change of the force on the first neighbor shells with increasing supercell size is less drastic now in the octahedral case and the directions of the forces on the second neighbor shells in $\mathrm{Pd}_{32} \mathrm{H}$ are found to be repulsive. These are very important improvements because the forces can be interpreted more consistently and the resulting force-dipole tensor turns out to be physically reasonable. (With the old data the attractive forces of the second neighbor shells had lead to negative force-dipole components.) Consequently, the forces on more remote atom shells can be used now in the construction of a $\mathrm{Pd}-\mathrm{H}$ pair potential,

TABLE VII. Absolute values of forces on $\mathrm{Nb}$ atoms at different neighbor shells around an octahedral $\mathrm{H}$ atom in $\mathrm{Nb}_{n} \mathrm{H}$ (in Ry/a.u.): $d / a$ is the distance between $\mathrm{Nb}$ and $\mathrm{H}$ atoms in units of the calculated equilibrium lattice constant of pure $\mathrm{Nb}, a=a_{0}(\mathrm{Nb})=6.11$ a.u. $=3.23 \AA$. Values marked by an asterisk indicate that these metal atoms are located on the Wigner-Seitz cell boundary of the hydrogen sublattice.

\begin{tabular}{cccccc}
\hline \multicolumn{5}{c}{$H$ at $O$ in $N b$} \\
Shell & $(1)$ & $(2)$ & $(3)$ & $(4)$ & $(5)$ \\
\hline$\frac{d}{a}$ & $\frac{1}{2}$ & $\frac{\sqrt{2}}{2}$ & $\frac{\sqrt{5}}{2}$ & $\frac{\sqrt{6}}{2}$ & $\frac{3}{2}$ \\
$\mathrm{NbH}$ & $0.0^{*}$ & & & & \\
$\mathrm{Nb}_{2} \mathrm{H}$ & $0.0^{*}$ & $0.0^{*}$ & & & \\
$\mathrm{Nb}_{4} \mathrm{H}$ & 0.147 & $0.0^{*}$ & & & \\
$\mathrm{Nb}_{8} \mathrm{H}$ & 0.151 & 0.021 & $0.0^{*}$ & & \\
$\mathrm{Nb}_{16} \mathrm{H}$ & 0.145 & 0.020 & $0.003^{*}$ & $0.004^{*}$ & $0.003^{*}$ \\
\hline \hline
\end{tabular}


as has been done in our recent model calculation for the stability of self-trapped states of $\mathrm{H}$ isotopes in $\mathrm{Pd} .{ }^{15}$

The components $A$ of the force-dipole tensors for $\mathrm{H}$ at the octahedral and tetrahedral sites are compiled in Tables IV and V, together with their individual contributions from the different neighbor shells of $\mathrm{Pd}$ atoms. These contributions are obtained by only summing over the equivalent $\mathrm{Pd}$ atoms of each shell. Traces $\operatorname{Tr} \mathcal{P}$ and nonzero off-diagonal components $C$ for $\mathrm{H}$ at all three sites are given in Table VI. Comparing the results for the different supercells we notice that the force-dipole contributions as well as the forces (cf. Tables I-III) on the inner atom shells are reasonably converged for the $\mathrm{Pd}_{32} \mathrm{H}$ supercells. The neighbor shells with $d / a \leq 1$ dominantly determine the force-dipole tensor. Contributions from shells with $d / a>1$, which are only present in the $\operatorname{Pd}_{32} \mathrm{H}$ cells, have to be considered with caution because the corresponding forces are well below our estimated limit of numerical significance. Taking them into account results in the values listed in parentheses in Table VI. Compared to the experimentally found trace of the tensor Tr $\mathcal{P}=3 A=10.5 \mathrm{eV},{ }^{20}$ the calculated value of $7.0 \mathrm{eV}$ (or $5.4 \mathrm{eV}$ when adding the spurious force contributions from the more remote shells) for the octahedral site agrees somewhat better than about $17 \mathrm{eV}$ and $18 \mathrm{eV}$ for the tetrahedral site and the saddle point, respectively, in accordance with the well confirmed knowledge of octahedral occupation of $\mathrm{H}$ in fcc $\mathrm{Pd}$.

Forces in the cubic $\mathrm{Nb}_{n} \mathrm{H}$ supercells $(n=1,2,4,8,16)$ have been calculated with the mixed-basis pseudopotential scheme mentioned above, continuing previous totalenergy calculations for the three smaller cells. ${ }^{21}$ The calculated magnitudes $\left|\mathbf{f}^{m}\right|$ are listed in Tables VII-IX for the $\mathrm{H}$ at the tetrahedral and octahedral sites in the bcc lattice and at the saddle point connecting two neighboring tetrahedral sites. With increasing cell size the forces on the first neighbor $\mathrm{Nb}$ shells converge satisfactorily. The decay of the forces towards the outer shells is somewhat slower than in $\mathrm{Pd}$, perhaps because of a weaker screening of the defect by the smaller number of valence electrons in the less closely packed bcc crystal. However, in $\mathrm{Nb}_{16} \mathrm{H}$ the forces on the shells with $d / a>1$ are again close to our estimated limit of numerical significance, as in $\mathrm{Pd}_{32} \mathrm{H}$.
TABLE VIII. Absolute values of forces on $\mathrm{Nb}$ atoms around a tetrahedral $\mathrm{H}$ atom in $\mathrm{Nb}_{n} \mathrm{H}$ (in $\mathrm{Ry} /$ a.u.). See the caption of Table VII.

\begin{tabular}{ccccc}
\hline \hline & \multicolumn{3}{c}{$\mathrm{H}$ at $T$ in $\mathrm{Nb}$} \\
Shell & $(1)$ & $(2)$ & $(3)$ & $(4)$ \\
\hline$\frac{d}{a}$ & $\frac{\sqrt{5}}{4}$ & $\frac{\sqrt{13}}{4}$ & $\frac{\sqrt{21}}{4}$ & $\frac{\sqrt{29}}{4}$ \\
$\mathrm{NbH}$ & $0.0^{*}$ & & & \\
$\mathrm{Nb}_{2} \mathrm{H}$ & $0.018^{*}$ & & & \\
$\mathrm{Nb}_{4} \mathrm{H}$ & 0.041 & & & \\
$\mathrm{Nb}_{8} \mathrm{H}$ & 0.050 & 0.008 & & \\
$\mathrm{Nb}_{16} \mathrm{H}$ & 0.051 & 0.005 & $0.001^{*}$ & $0.003^{*}$ \\
\hline \hline
\end{tabular}

The tensor components of the force dipole for the three interstitial sites in the three larger supercells are listed in Table X. As in the case of $\mathrm{Pd}$ they are dominated by the forces of the shells with $d / a \leq 1$. Contributions from the further shells in $\mathrm{Nb}_{16} \mathrm{H}$ should be taken with caution again because there the uncertainty in the values of the small force components is enlarged for the values of the force-dipole tensor by the multiplication with the increasing distance components. Results by including these are given in parentheses in Table $\mathrm{X}$. With respect to the experimental observation of the trace $\operatorname{Tr} \mathcal{P}=2 A+B=10.1 \pm 0.3 \mathrm{eV}$ and the anisotropy $\frac{3|A-B|}{2 A+B} \leq 0.06$ (Ref. 4) of the force-dipole tensor we find astonishingly good quantitative agreement of the calculated values of about $11 \mathrm{eV}$ and 0.1 for the tetrahedral site. For the other two sites both the traces and anisotropies are considerably larger.

To summarize, we have calculated the forces on $\mathrm{Pd}$ and $\mathrm{Nb}$ atoms surrounding a $\mathrm{H}$ atom on different interstitial sites in fcc $\mathrm{Pd}_{n} \mathrm{H}$ and bcc $\mathrm{Nb}_{n} \mathrm{H}$ supercells from first principles. We find that the forces decay reasonably quickly within the range of one lattice constant. In the comparison of the force-dipole components with experimental results we find the best agreement for the experimentally well confirmed occupied octahedral and tetrahedral sites in $\mathrm{Pd}$ and $\mathrm{Nb}$, respectively, both for the traces of the force-dipole tensor and in the bcc metal for the very small anisotropy. Based on these results we think that

TABLE IX. Absolute values of forces on a $\mathrm{H}$ atom located at a saddle point between two tetrahedral sites in $\mathrm{Nb}_{n} \mathrm{H}$ and on the $\mathrm{Nb}$ atoms around it (in Ry/a.u.). See the caption of Table VII. The numbers separated by a vertical bar denote forces on Pd atoms on symmetrically inequivalent positions, but with the same distance to the $\mathrm{H}$ atom.

\begin{tabular}{|c|c|c|c|c|c|c|c|c|}
\hline \multicolumn{9}{|c|}{$\mathrm{H}$ at $S$ in $\mathrm{Nb}$} \\
\hline Shell & $\mathrm{H}$ & $(1)$ & $(2)$ & (3) & (4) & $(5)$ & $(6)$ & $(7)$ \\
\hline$\frac{d}{a}$ & 0 & $\frac{\sqrt{18}}{8}$ & $\frac{\sqrt{34}}{8}$ & $\frac{\sqrt{50}}{8}$ & $\frac{\sqrt{66}}{8}$ & $\frac{\sqrt{82}}{8}$ & $\frac{\sqrt{98}}{8}$ & $\frac{\sqrt{114}}{8}$ \\
\hline $\mathrm{NbH}$ & 0.0 & 0.0 & & & & & & \\
\hline $\mathrm{Nb}_{2} \mathrm{H}$ & 0.021 & $0.076 \mid 0.026^{*}$ & & & & & & \\
\hline $\mathrm{Nb}_{4} \mathrm{H}$ & 0.022 & $0.085 \mid 0.087$ & $0.004^{*}$ & & & & & \\
\hline $\mathrm{Nb}_{8} \mathrm{H}$ & 0.021 & $0.077 \mid 0.086$ & 0.015 & 0.006 & $0.000^{*}$ & & & \\
\hline $\mathrm{Nb}_{16} \mathrm{H}$ & 0.020 & $0.087 \mid 0.087$ & 0.013 & 0.004 & 0.003 & $0.001^{*}$ & $0.003^{*}$ & $0.003^{*} \mid 0.004^{*}$ \\
\hline
\end{tabular}


TABLE X. Traces (in eV), anisotropies, and nonzero off-diagonal components $C$ (in $\mathrm{eV}$ ) of the force-dipole tensors for $\mathrm{H}$ at the octahedral and tetrahedral sites and the saddle point in $\mathrm{Nb}$. Values in parentheses contain force contributions from shells with $d / a>1$.

\begin{tabular}{cccccccc}
\hline & \multicolumn{2}{c}{$\mathrm{H}$ at $O$} & \multicolumn{4}{c}{$\mathrm{H}$ at $T$} & $\mathrm{H}$ at $S$ \\
& $\operatorname{Tr} \mathcal{P}$ & $\frac{3|A-B|}{2 A+B}$ & $\operatorname{Tr} \mathcal{P}$ & $\frac{3|A-B|}{2 A+B}$ & $\operatorname{Tr} \mathcal{P}$ & $\frac{3|A-B|}{2 A+B}$ & $C$ \\
\hline $\mathrm{Nb}_{4} \mathrm{H}$ & 12.2 & 3.0 & 7.6 & 0.4 & 11.5 & 1.2 & 2.4 \\
$\mathrm{Nb}_{8} \mathrm{H}$ & 17.4 & 2.4 & 11.6 & 0.0 & 13.3 & 0.8 & 1.4 \\
$\mathrm{Nb}_{16} \mathrm{H}$ & $16.8(13.3)$ & $1.7(2.0)$ & $10.9(10.5)$ & $0.1(0.2)$ & $13.3(13.7)$ & $0.8(0.5)$ & $1.8(2.8)$ \\
\hline \hline
\end{tabular}

the small anisotropy of the force-dipole tensor in $\mathrm{Nb}$ can be quantitatively explained by static forces of reasonably short range. However, since the small anisotropy of $\mathcal{P}$ is only one of several arguments in favor of a dynamical model, we do not want to rule out its necessity to explain more properties of the local defect structure of $\mathrm{H}$ isotopes in $\mathrm{Nb}$, as proposed by Dosch et al. ${ }^{8}$ and supported by Wahnström and $\mathrm{Li}^{9}{ }^{9}$
The Ames Laboratory is operated for the U.S. Department of Energy by the Iowa State University under Contract No. W-7405-ENG-82. This work was supported in part by the Director of Energy Research, Office of Basic Energy Sciences, including a grant of computer time on the Cray computers at the NERSC in Livermore, California, and by the NATO Collaborative Research Grant No. 910439 .
${ }^{1}$ Y. Fukai, The Metal-Hydrogen System (Springer, Berlin, 1993).

${ }^{2}$ Hydrogen in Metals I, edited by G. Alefeld and J. Völkl (Springer, Berlin, 1978).

${ }^{3} \mathrm{H}$. Wagner, in Hydrogen in Metals I (Ref. 2), Chap. 2.

${ }^{4} \mathrm{H}$. Peisl, in Hydrogen in Metals I (Ref. 2), Chap. 3.

${ }^{5}$ H.-D. Carstanjen, Phys. Status Solidi A 59, 11 (1980).

${ }^{6}$ H. Kanzaki, J. Phys. Chem. Solids 2, 24 (1957).

${ }^{7}$ Point Defects in Metals I, edited by G. Leibfried and N. Breuer (Springer, Berlin, 1978).

${ }^{8}$ H. Dosch and J. Peisl, Phys. Rev. Lett. 56, 1385 (1986); H. Dosch, J. Peisl, and B. Dorner, Phys. Rev. B 35, 3069 (1987); H. Dosch, F. Schmid, P. Wiethoff, and J. Peisl, ibid. 46, 55 (1992).

${ }^{9}$ G. Wahnström and Y. Li, Phys. Rev. Lett. 71, 1031 (1993).

${ }^{10} \mathrm{P}$. Hohenberg and W. Kohn, Phys. Rev. 136, B864 (1964); W. Kohn and L. J. Sham, ibid. 140, A1133 (1965).

${ }^{11}$ C. Elsässer, K. M. Ho, C. T. Chan, and M. Fähnle, J. Phys.
Condens. Matter 4, 5207 (1992).

${ }^{12}$ M. Weinert and J. W. Davenport, Phys. Rev. B 45, 13709 (1992).

${ }^{13}$ C. Elsässer, M. Fähnle, C. T. Chan, and K. M. Ho, Phys. Rev. B 49, 13975 (1994).

${ }^{14}$ A. Mällo and U. Yxklinten, Z. Phys. B 83, 213 (1991).

${ }^{15}$ H. Krimmel, L. Schimmele, C. Elsässer, and M. Fähnle, J. Phys. Condens. Matter (to be published).

${ }^{16}$ S. G. Louie, K. M. Ho, and M. L. Cohen, Phys. Rev. B 19, 1774 (1979).

${ }^{17}$ C. L. Fu and K. M. Ho, Phys. Rev. B 28, 5480 (1983).

${ }^{18}$ K. M. Ho, C. L. Fu, and B. N. Harmon, Phys. Rev. B 28, 6687 (1983).

${ }^{19}$ K. M. Ho, C. Elsässer, C. T. Chan, and M. Fähnle, J. Phys. Condens. Matter 4, 5189 (1992).

${ }^{20} \mathrm{~K}$. W. Kehr, in Hydrogen in Metals I (Ref. 2), Chap. 8.

${ }^{21}$ C. Elsässer, M. Fähnle, K. M. Ho, and C. T. Chan, Physica B 172, 217 (1991). 